\title{
Day-7 or day-90 modified Rankin scale score: what is the best measure of outcome after thrombolysis in ischemic stroke?
}

\author{
Jean-Marc Bugnicourt • Olivier Godefroy
}

Published online: 2 October 2012

(C) Springer Science+Business Media New York 2012

\section{To the Editor}

We read the article by Cappellari and colleagues [1] with great interest. The authors suggest that, in ischemic stroke patients, the best time point to assess final functional outcome after intravenous thrombolysis, using modified Rankin scale (mRS) score [2], should be 7 days instead of the well established 3 or 6 months delay. This earlier evaluation of the thrombolysis direct effect may particularly improve primary outcome measurement in acute stroke trials, as evaluation usually suffers from missing data on 3-month mRS score. This comment follows the results of a clinical study published recently [3]. However, the reduction of missing data may be counterbalanced by decrease reliability of grading.

The main point concerns the mRS, which is effectively the most widely used disability scale in clinical practice and in contemporary stroke trials. However, criteria defining each stage in the conventional mRS have been defined for non hospitalized patients. Several new versions of the Rankin scale, incorporating a formal structured interview, have been very recently proposed to increase reliability of Rankin grading $[4,5]$. These revised versions usually improve reliability and sensitivity, which increases the mean grading of disability especially for grade 1-3. The following two examples are given to illustrate our position. First, 1 week after a stroke (even of moderate

\section{J.-M. Bugnicourt ( $\square)$}

Service de Neurologie, CHU Amiens, Victor Pauchet,

80054 Amiens Cedex 1, France

e-mail: bugnicourt.jean-marc@chu-amiens.fr

J.-M. Bugnicourt · O. Godefroy

Laboratoire de Neurosciences Fonctionnelles et Pathologies,

Amiens University Hospital, Amiens, France severity), most patients have usually full employment restriction and thus a mRs of 2 should be graded (defined as a limitation in participation in usual roles), even if the patient has no symptoms at all. Second, it is not possible to score Rankin scale in the context of hospitalization as it requires to grade to ability perform activities of daily living such as "assistance for preparing a simple meal?" or "doing household chores?".

This shows that an early assessment does not reflect real life and long term abilities. This emphasizes that improvement of the assessment of outcome in stroke will be mainly provided by systematic use of a structured interview to assign grades on the mRS.

\section{References}

1. Cappellari M, Moretto G, Bovi P (2012) Day-7 modified Rankin Scale score as the best measure of the thrombolysis direct effect on stroke? J Thromb Thrombolysis. doi:10.1007/s11239-012-0805-8

2. van Swieten JC, Koudstaal PJ, Visser MC, Schouten HJ, van GJ (1988) Interobserver agreement for the assessment of handicap in stroke patients. Stroke 19:604-607

3. Ovbiagele B, Saver JL (2010) Day-90 acute ischemic stroke outcomes can be derived from early functional activity level. Cerebrovasc Dis 29:50-56

4. Wilson JTL, Hareendran A, Grant M, Baird T, Schulz UGR, Muir KW, Bone I (2002) Improving the assessment of outcomes in stroke: use of a structured interview to assign grades on the modified Rankin Scale. Stroke 33:2243-2246

5. Saver JL, Filip B, Hamilton S, Yanes A, Craig S, Cho M, Conwit R, Starkman S, for the FAST-MAG Investigators and Coordinators (2010) Improving the reliability of stroke disability grading in clinical trials and clinical practice. The Rankin focused assessment. Stroke 41:992-995 